\title{
Study and Characterization of Indium Oxynitride Photoconductors
}

\author{
M. Sparvoli ${ }^{a *}$, R. D. Mansano ${ }^{a}$, J. F. D. Chubaci ${ }^{b}$ \\ ${ }^{a}$ Laboratório de Sistemas Integráveis, Departamento de Engenharia de Sistemas Eletrônicos, \\ Escola Politécnica, Universidade de São Paulo - USP, Av. Prof. Luciano Gualberto, \\ trav. 3, 153, CEP 05508-900, São Paulo, SP, Brasil \\ ${ }^{b}$ Instituto de Física, Universidade de São Paulo - USP, CEP 05314-970, São Paulo, SP, Brasil
}

Received: June 9, 2013; Revised: November 7, 2013

\begin{abstract}
Multifunctional materials are a new class of thin films and coatings. These materials show interesting characteristics for application in many scientific areas, in special electronic and photonic technologies. These characteristics include sensitivity for thermal, light, mechanical, chemical and other influences, high resistivity, high electrical isolation and transparence in visible range. Recently it was obtained a new oxide type that combines oxygen, nitrogen and indium: the indium oxynitride. In this work, we study the deposition of indium oxynitride by reactive sputtering for application in photoconductor sensors. The deposition processes were performed in a home build magnetron sputtering system, using a four-inch pure In target, nitrogen and oxygen as process gases. The pressure was kept constant at 10 mtorr and the RF power (13.56 MHz) was constant at $250 \mathrm{~W}$. The photoconductors were made with these thin films. The photoelectric detectors were analyzed by $\mathrm{IxV}$ (current versus voltage) analyses. The IxV analysis presented a low leakage current $\left(10^{-8} \mathrm{~A}\right)$. The photoelectric effect was observed from the difference between the case with emitted light and dark currents. It increased around 140 times, under illumination of a halogen lamp. The Hall Effect measurements indicated that the films were n-type semiconductors. The increase in the oxygen concentration added in the plasma, promoted the change in the character of these thin films from conductor to semiconductor material.
\end{abstract}

Keywords: indium oxynitride, optoelectronic and photonic sensors, photo detectors, thin film sensors

\section{Introduction}

The area of optoelectronics has seen substantial progress in recent years. Especially with the discovery of new materials that can be used as light sensors. Indium oxynitride (InNO) can be considered as a new material with properties between semiconductor indium nitride ( $\mathrm{InN}$ ) and insulator indium oxide $\left(\mathrm{In}_{2} \mathrm{O}_{3}\right)^{[1,2]}$.

InNO is a semitransparent material of pale yellow color and has a wide band gap in the range 3.4-3.6 eV (nearest indium oxide), which makes it an excellent candidate to be employed in photo sensors in the ultraviolet region. Furthermore, it can be used as material for solar cells fabrication $^{3}$. This ternary alloy belongs to a new class of materials with optical, mechanical and electrical properties which are potentially interesting for industrial applications ${ }^{4}$. Numerous properties of the InNO, such as the refractive index and the photoelectric effect intensity, vary according to the proportion of oxygen and nitrogen contained in the formed film.

In this work we study the deposition of indium oxynitride by reactive sputtering for application in photoconductor sensors. The photoelectric detectors can be divided into three classes: the photoconductors, photovoltaic and photo emitters. The photoconductive and photovoltaic detectors are directly based on semiconductors in which photons are

*e-mail: marinsparvoli@yahoo.com.br absorbed by the semiconductor material, producing electronhole pairs, which are separated by an electric field. These detectors use the photoelectric effect (internal), where the photon energy is high enough to carry the electrons to the conduction band of the semiconductor material.

The photoconductor on the other hand, is essentially a resistor sensitive to radiation, consisting of two ohmic contacts. A photon with energy hv, higher than the band gap energy is absorbed to produce an electron-hole pair, consequently changing the electrical conductivity of the semiconductor. In most cases, the change in conductivity is measured through electrodes fixed on the semiconductor surface. For a material with low resistance, the photoconductor is normally operated in a constant current circuit mode. Examples are the photoconductors made of GaAs, GaN and AlGaN junctions.

\section{Materials and Methods}

\subsection{Deposition process}

In this work, silicon wafers with resistivity in the range of 10-20 Ohm $\mathrm{cm}$, three inch diameter, $280 \mathrm{~mm}$ thick, p-type, (100) were used.

Initially, a cleaning process $\left(4 \mathrm{H}_{2} \mathrm{SO}_{4}+1 \mathrm{H}_{2} \mathrm{O}_{2}\right)$ followed by $\mathrm{HF}$ dipping (2\%), was performed. The deposition of 
the indium oxynitride films was performed in magnetron sputtering system using an indium pure target. Five series of thin films were deposited with oxygen $(10 \%, 20 \%, 50 \%$, $80 \%$ and $85 \%$ ) and nitrogen.

\subsection{Hall effect measurements}

This technique enables one to obtain the values of charge density, resistivity, sheet resistance, mobility and carrier type (n-type or p-type). The measurements in this study were made at the Instituto de Física da Universidade de São Paulo with equipment from MMR technologies (Hall Van de Pauw Controller H50).

\subsection{I-V measurements}

In this work, we manufactured light-sensitive ohmic photoconductors using aluminum as the metal contact deposited on the InNO thin film (Figure 1). The potential difference is applied on the two aluminum contacts $(0.5 \mathrm{~cm}$ diameter) and the halogen lamp light was focused in the film between them. In the photoconductor fabrication, aluminum circle contacts were deposited at the front side of the wafer by evaporation in order to obtain $300 \mathrm{~nm}$ thick layer. At the backside of the wafer, a 500 $\mathrm{nm}$ thick aluminum film was evaporated. Measurements were made with and without the presence of light from the halogen lamp.

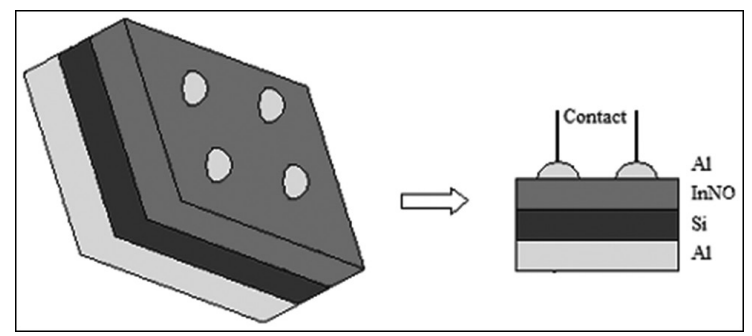

Figure 1. Design of photoconductor used in this study.

Table 1. Hall effect measurements.

\begin{tabular}{ccc}
\hline Sample & $\begin{array}{c}\text { Resistivity } \\
(\Omega \text { cm })\end{array}$ & Type \\
\hline $10 \% \mathrm{O}_{2}$ & $1.05 \times 10^{-2}$ & $\mathrm{~N}$ \\
$20 \% \mathrm{O}_{2}$ & 0.52 & $\mathrm{~N}$ \\
$50 \% \mathrm{O}_{2}$ & 0.76 & $\mathrm{~N}$ \\
$80 \% \mathrm{O}_{2}$ & $3.02 \times 10^{2}$ & $\mathrm{~N}$ \\
$85 \% \mathrm{O}_{2}$ & $4.76 \times 10^{4}$ & $\mathrm{~N}$ \\
\hline
\end{tabular}

\section{Results and Discussion}

The electrical measurements were carried out at the Instituto de Física da USP with a MMR technologies (Hall Van de Pauw Controller H50) equipment, in room temperature.

The results obtained from the Hall effect measurements (Table 1) show in general that the resistivity of the InNO films deposited on $\mathrm{Si}$ is very high (using $\mathrm{InN}$ values as reference: 1.6 to $4 \mathrm{~mW} . \mathrm{cm}^{[3]}$ ). It can be associated with the photoconductor IxV analysis that shows a low leakage current (Tables 2 and 3).

The indium nitride is an n-type semiconductor due to the high amount of defects in its structure ${ }^{4,5}$. When an alloy as the indium oxynitride is deposited, some nitrogen bonds are eventually replaced by oxygen. The defects remain and the semiconductor remains n-type.

When oxygen is added, the resistivity increases gradually. Indium nitride thin films are semiconductors that are closest to conductive materials due to its low resistivity, a value equivalent to $3 \times 10^{-3} \Omega \mathrm{cm}^{[6,7]}$.

It is evident that the InNO deposited with $10 \%$ oxygen has resistivity $\left(1.1 \times 10^{-2} \Omega \mathrm{cm}\right)$ closer to $\mathrm{InN}$. In other hand, the InNO deposited with $85 \%$ oxygen has a much higher resistivity $\left(4.8 \times 10^{4} \Omega \mathrm{cm}\right)$, approaching of the $\mathrm{InO}$ resistivity, which is an insulator.

The intensity of the photoelectric effect was observed from the difference of case with light on and dark current values. The results are shown in Figure 2 to Figure 6.

All manufactured devices were analyzed. It was verified that the photoconductor that showed notable results in terms of electrical measurement $(\mathrm{IxV})$ and photoelectric effect was the one deposited with InNO thin film, with oxygen concentration of $20 \%$ and $250 \mathrm{~W}$ RF power on p-type silicon substrate. The added oxygen forms the indium oxynitride thin film and establishes links with metallic indium, thereby decreasing the carrier concentration and leakage current (dark current), which promotes the increase of the photoelectric effect ${ }^{8}$. However, high concentrations of oxygen decrease conductivity and sensor sensitivity.

On Table 2, it can be observed that this sample was the one with the lowest dark current, indicating low leakage current $\left(3.74 \times 10^{-8} \mathrm{~A}\right)$. In addition to this fact, the observed photoelectric effect intensity is the biggest for among all the samples (increased 139.8 times).

In Table 3 we present the results of light and dark currents in the presence of negative electric field. With these data it was verified that the graphs are in fact symmetric, which indicates an ohmic behavior.

Table 2. Electrical measurement (I-V).

\begin{tabular}{lcccc}
\hline Sample & Light current $(\mathbf{A})$ & Dark current $(\mathbf{A})$ & Increasing (times) & Position (V/cm) \\
\hline $10 \% \mathrm{O}_{2}$ & $1.37 \times 10^{-5}$ & $1.12 \times 10^{-7}$ & 122.4 & $5.5 \times 10^{5}$ \\
$20 \% \mathrm{O}_{2}$ & $5.23 \times 10^{-6}$ & $3.74 \times 10^{-8}$ & 139.8 & $5.5 \times 10^{5}$ \\
$50 \% \mathrm{O}_{2}$ & $3.20 \times 10^{-6}$ & $9.31 \times 10^{-8}$ & 34.4 & $5.5 \times 10^{5}$ \\
$80 \% \mathrm{O}_{2}$ & $7.89 \times 10^{-7}$ & $5.10 \times 10^{-8}$ & 15.5 & $5.5 \times 10^{5}$ \\
$85 \% \mathrm{O}_{2}$ & $3.82 \times 10^{-7}$ & $2.64 \times 10^{-8}$ & 14.5 & $5.5 \times 10^{5}$ \\
\hline
\end{tabular}


Table 3. Electrical measurement (I-V).

\begin{tabular}{ccccc}
\hline Sample & Light current (A) & Dark current (A) & Increasing (times) & Position (V/cm) \\
\hline $10 \% \mathrm{O}_{2}$ & $1.22 \times 10^{-5}$ & $1.20 \times 10^{-7}$ & 101.7 & $-5.5 \times 10^{5}$ \\
$20 \% \mathrm{O}_{2}$ & $3.87 \times 10^{-6}$ & $3.86 \times 10^{-8}$ & 100.3 & $-5.5 \times 10^{5}$ \\
$50 \% \mathrm{O}_{2}$ & $2.45 \times 10^{-6}$ & $8.15 \times 10^{-8}$ & 30.1 & $-5.5 \times 10^{5}$ \\
$80 \% \mathrm{O}_{2}$ & $1.02 \times 10^{-6}$ & $2.96 \times 10^{-8}$ & 34.5 & $-5.5 \times 10^{5}$ \\
$85 \% \mathrm{O}_{2}$ & $5.00 \times 10^{-7}$ & $4.22 \times 10^{-8}$ & 11.9 & $-5.5 \times 10^{5}$ \\
\hline
\end{tabular}

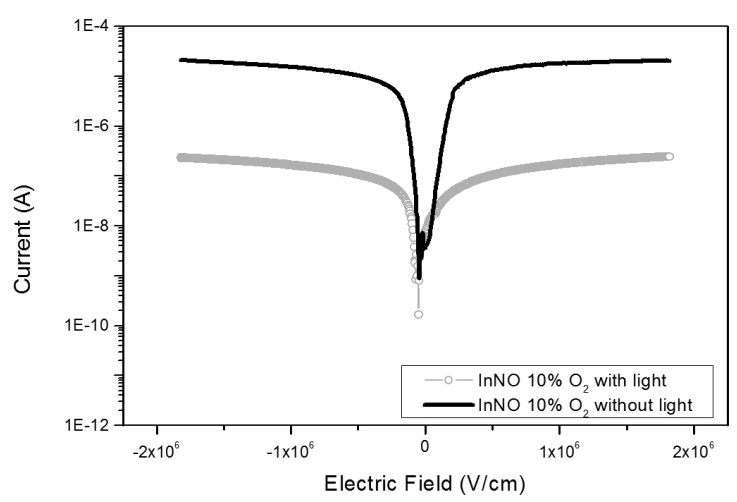

Figure 2. Current as a function of Electric Field for InNO $10 \% \mathrm{O}_{2}$ with light and without light.

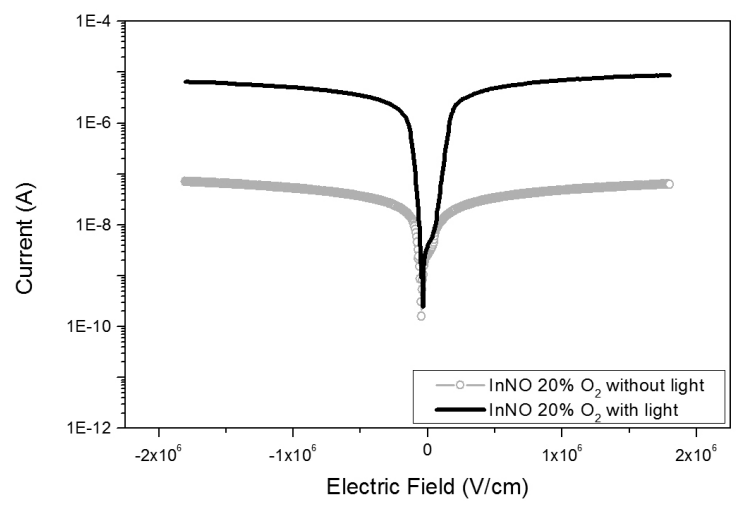

Figure 3. Current as a function of Electric Field for InNO 20\% $\mathrm{O}_{2}$ with light and without light.

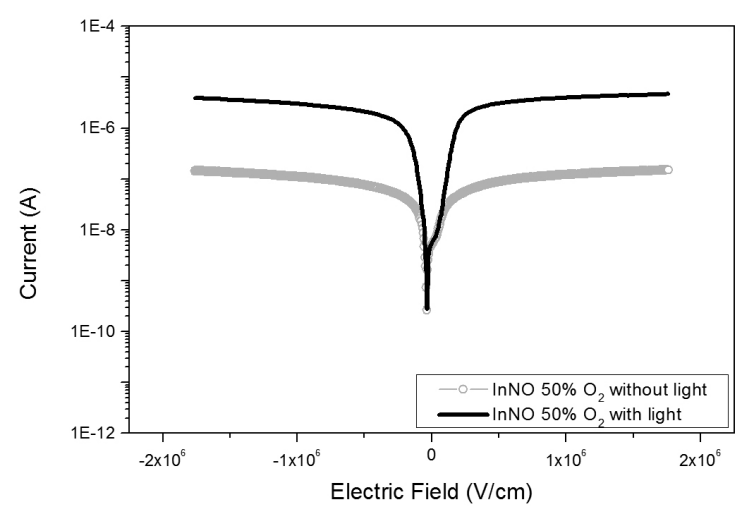

Figure 4. Current as a function of Electric Field for InNO 50\% $\mathrm{O}_{2}$ with light and without light.

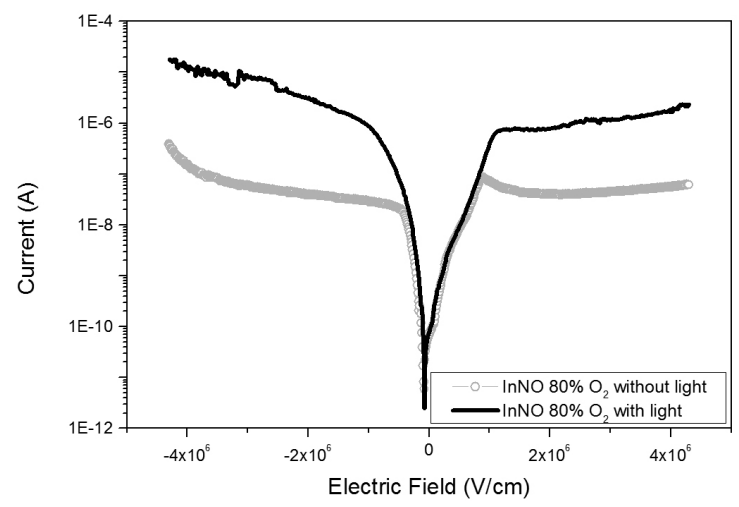

Figure 5. Current as a function of Electric Field for InNO $80 \% \mathrm{O}_{2}$ with light and without light.

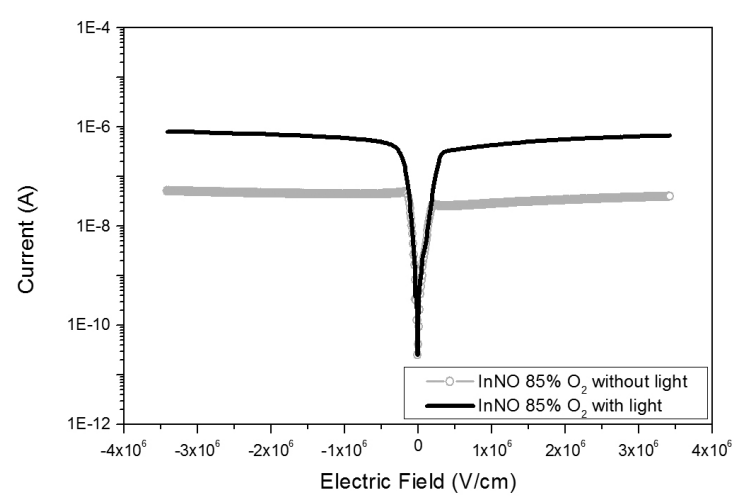

Figure 6. Current as a function of Electric Field for InNO $85 \% \mathrm{O}_{2}$ with light and without light.

The fact is that the aluminum acts as an ohmic contact in these samples. Therefore, it is being used as part of the sensors.

Incorporation of oxygen to the film reduces the conductivity and, therefore, material defects. Addition of oxygen facilitates the formation of indium oxynitride thin film, thereby decreasing the carrier concentration and leakage current, eventually enhancing the photoelectric effect in low concentrations of this element in deposition process of thin film.

With these satisfactory results, it is possible to use such devices in many areas. These sensors may be used, for example, as photosensor for aerosol detection, after been calibrated with the assistance of a pyranometer. It can also still be used in biomedical and optical sensors. 


\section{Conclusions}

The films produced with the addition of different concentrations of oxygen form the indium oxynitride, a ternary alloy with promising characteristics for application in the microelectronics area. These films showed photoelectric effect with high intensities, especially the thin film deposited with InNO $20 \%$ oxygen.

There are evidences that the incorporation of oxygen to the film reduces the conductivity, which may mean that there was a reduction of defects in that material. The added oxygen forms the indium oxynitride thin film and establishes links with metallic indium, thereby decreasing the carrier

\section{References}

1. Miyake A, Masubuchi Y, Takeda T and Kikkawa S. Indium and gallium oxynitrides prepared in the presence of $\mathrm{Zn}^{2+}$ by ammonolysis of the oxide precursors obtained via the citrate route. Materials Research Bulletin. 2009; 45(4):505-508. http:// dx.doi.org/10.1016/j.materresbull.2009.10.008

2. Zervos M, Tsokkou D, Pervolaraki M and Othonos A. Low Temperature Growth of $\operatorname{In}_{2} \mathrm{O}_{3}$ and InN Nanocrystals on $\mathrm{Si}(111)$ via Chemical Vapour Deposition Based on the Sublimation of $\mathrm{NH}_{4} \mathrm{Cl}$ in In. Nanoscale Research Letters. 2008; 4(6):491-497. http://dx.doi.org/10.1007/s11671-009-9266-1

3. Sungthong A, Porntheeraphat S, Poyai A and Nukeaw J. An extreme change in structural and optical properties of indium oxynitride deposited by reactive gas-timing RF magnetron sputtering. Applied Surface Science. 2008; 254(23):7950-7954. http://dx.doi.org/10.1016/j.apsusc.2008.04.038

4. Ko TS, Chu CP, Chen HG, Lu TC, Kuo HC and Wang SC. Observation of strong red photoluminescence with broadband concentration and leakage current (dark current), which promotes the increase of the photoelectric effect. However, high concentrations of oxygen decrease conductivity and sensor sensitivity.

\section{Acknowledgments}

The authors would like to thank to the Laboratório de Sistemas Integráveis and Instituto de Física da Universidade de São Paulo for the measurements, to Mr. Nelson Ordonez for technical support and Fapesp, CNPq for financial support.

in indium oxynitride nanoparticles. Journal of Vacuum Science and Technology A. 2006; 24:1332-1335. http://dx.doi. org/10.1116/1.2198863

5. Jones RE, Yu KM, Li SX, Walukiewicz W, Ager JW, Haller EE et al. Evidence for p-type doping of InN. Physical Review Letters. 2006; 96(12):125505. http://dx.doi.org/10.1103/ PhysRevLett.96.125505

6. Davis RF. III-V Nitrides for electronic and Optoelectronic applications. Proceedings of the IEEE. 1991; 79:702-712.

7. Miura N, Ishii H, Yamada A, Konagai M, Yamauchi Y and Yamamoto A. Anomalous electrical characteristics of epitaxial $\mathrm{InN}$ film having a high electron concentration at very low temperature. Japanese Journal of Applied Physics. 1997; 36(2):256-259. http://dx.doi.org/10.1143/ JJAP.36.L256

8. Sparvoli M, Mansano RD, Zambom LS and Chubaci JFD. Optical and electrical properties of sputtered InNO thin films. Physica Status Solidi C. 2012; 9(6):1384-1387. 